\title{
Síndrome da Morte Súbita Infantil (SMSI): aspectos acerca das principais causas e as formas de prevenção
}

\author{
Maria Eliana Pierre Martins ${ }^{\text {; }}$ Liromaria Maria de Amorim ${ }^{2}$; Micaelle Nayara Dias Rodrigues ${ }^{3}$; \\ Danielly Gonçalves Sombra Lima ${ }^{4}$; Jamacir Ferreira Moreira ${ }^{5}$
}

\begin{abstract}
Resumo: O presente artigo objetiva refletir aspectos relacionados a Síndrome da Morte Súbita Infantil (SMSI). A SMSI é uma expressão usada para se referir a uma morte repentina e inesperada, na maioria dos casos inexplicável, que pode acometer os bebês no seu primeiro ano de vida. Trata-se de uma das maiores causas de morte infantil, ocupando inclusive a oitava posição nos países desenvolvidos. Em países como o Brasil, não existe uma coleta de dados que seja suficiente para uma avaliação das influências socioculturais ou geográficas quanto ao desenvolvimento da enfermidade. Ações educativas direcionadas às gestantes, mães, pais e cuidadores, podem ajudar a reduzir os casos de mortalidade subida infantil.
\end{abstract}

Palavras-Chave: Morte súbita. Mortalidade infantil. Políticas públicas.

\section{Sudden Infant Death Syndrome (SIDS): Issues regarding the main causes and Prevention.}

\begin{abstract}
This article aims to reflect aspects related to Sudden Infant Death Syndrome (SIDS). SIDS is an expression used to refer to a sudden and unexpected death, in most cases unexplained, that can affect infants in their first year of life. It is one of the major causes of child deaths, occupying the eighth position in developed countries. In countries like Brazil, there is no data collection that is sufficient for an evaluation of sociocultural or geographic influences regarding the development of the disease. Educational actions aimed at pregnant women, mothers, fathers and caregivers can help reduce cases of infant mortality.
\end{abstract}

Keywords: Sudden death. Child mortality. Public policy.

\footnotetext{
${ }^{1}$ Graduada em Medicina pela Universidade de Pernambuco. Doutoranda em Ciências da Saúde pela Faculdade do ABC Paulista. Mestrado em Saúde da Criança e do Adolescente pela Universidade Estadual do Cear. Residencia Medica em Pediatria no Hospital Barão de LucenaINAMPS. Especialização em Medicina da Família e Comunidade na Universidade Federal do Ceará-UFC(2008). Atualmente é Professor Adjunto e preceptora da Residência medica de pediatria - Faculdade de Medicina-Universidade Federal do Cariri e Médica Pediatra da Prefeitura Municipal do Crato. Contato: epierremartins@hotmail.com

${ }^{2}$ Graduação em Saúde Pública e Pedagogia, pela Universidade Regional do Cariri - URCA; Especialização em Educação Infantil e Ensino Fundamental pela Faculdade de Juazeiro do Norte - FJN; Especialização em Educação Infantil pela Universidade Regional do cariri - URCA. Contato: lirinhamor@gmail.com.

${ }^{3}$ Graduação em Administração pelo centro Universitário Doutor Leão Sampaio - UNILEÃO. Especialização em Recursos Humanos pela Faculdade de Juazeiro do Norte - FJN. Contato: micaellenayara@ hotmail.com;

${ }^{4}$ Graduação em fisioterapia pela Universidade Estadual da Paraíba. Pós-graduação latu sensu em fisioterapia hospitalar pela Faculdade Integrada do Ceará - FIC; Pós-graduação em Saúde da Família pela FIP - Faculdade Integrada de Patos; Pós graduação em docência do ensino superior pela Faculdade Leão Sampaio. Acadêmica do curso de Medicina da Universidade Estácio - FMJ. daniellygsombra@ hotmail.com.

${ }^{5}$ Médico Ortopedista e Traumatologista Membro da Sociedade Brasileira de Ortopedia e Traumatologia (SBOT). Membro da Associação Brasileira Ortopedica de Osteometabolismo (ABOOM) Titulo de Especialista em Ortopedia e Traumatologia pela Sociedade Brasileira de Ortopedia e Traumatologia (SBOT) Título de Especialista em Ortopedia e Traumatologia pelo Ministerio da Educação e Cultura (MEC) Título de Especialista em Ortopedia e Traumatologia pelo Conselho Regional de Médicina da Paraiba (CRM-PB) Medico Assistente do Hospital Regional do Cariri (HRC). Contato: jamacir@jamacir.com.br.
} 


\section{Introdução}

A Síndrome da Morte Súbita Infantil (SMSI), é um dos principais fatores de morte de crianças aparentemente saudáveis, que ocorre, em regra, durante o sono do bebê e quando este possui até, em média, 356 dias de vida. Ademais, já se identificou que a SMSI ocorre com mais frequência em bebês prematuros e que nasçam com baixo peso - com menos de $2,5 \mathrm{~kg}$-, visto que estes desenvolvem tardiamente suas atividades de controle cerebral, estão mais propensos a desenvolver enfermidades e são mais facilmente acometidos pela desnutrição.

Os fatores fisiopatológicos indicados como causadores da SMSI, possuem uma origem multifatorial, dentre os quais encontra-se: prematuridade, baixo peso, forma e local onde a criança dorme, possuir de 2 a 4 meses de idade, ser do sexo masculino, e a mãe adolescente, com baixo grau de escolaridade ou fumante, como principais fatores de risco.

No Brasil, a síndrome ocupa a lista mortes que poderiam ser evitadas com a devida intervenção do sistema de saúde pública, inclusive, de acordo com o Departamento de Informática do SUS (DATASUS), dos óbitos por morte súbita infantil ocorridos no ano de 2012, aproximadamente $27 \%$ foram na região Nordeste (MINISTÉRIO DA SAÚDE, 2012), o que demonstra a necessidade do desenvolvimento de campanhas educativas no país com o objetivo de orientar profissionais da saúde e a população acerca das medidas preventivas adequadas.

Raros são os estudos acerca do tema, o que limita a possibilidade de realizar análises sobre a temática, no entanto, sua importância revela a necessidade de incentivo ao desenvolvimento de estudos acerca da síndrome, bem como a proposição e implemento de políticas públicas preventivas que se adequem as necessidades locais, levando em conta as peculiaridades socioeconômicas, culturais e geográficas.

\section{Breves considerações acerca da Síndrome da Morte Súbita Infantil e sua incidência no Brasil}

A Síndrome da Morte Súbita Infantil (SMSI), trata-se do falecimento inesperado de uma criança, de forma assintomática ou com ínfimos sintomas ocorridos com menos de 24 horas de 
duração (VICTORA ET AL, 1987, p. 490-496), sendo assim, consiste em diagnóstico em que um bebê, que aparentemente estava saudável, morre repentinamente e sem a devida explicação, em que nem a autópsia, tampouco os médicos, são capazes de explicar a causa da morte.

A SMSI, ocupa a primeira causa de mortalidade pós-neonatal na atualidade, inclusive nos países desenvolvidos, e, a oitava enfermidade que mais causa mortalidade infantil. Sua taxa de incidência alcança 90 mortes no Japão, 41 no Reino Unido, 57 nos Estados Unidos, 80 na Nova Zelândia, a cada 1000 vivos. Contudo, no Brasil ainda não existem dados específicos acerca da mortalidade infantil por morte súbita.

Esse fenômeno passou a ocupar o centro das atenções nas últimas décadas, em razão da diminuição das taxas de mortalidade infantil, proveniente de melhoras na assistência médica do país, bem como a descoberta das melhores formas de prevenção. Especificamente no Brasil, os índices de mortalidade infantil têm a diminuído de forma contínua, abarcando uma redução $39 \%$ entre os anos de 2000 e 2010, o que significa uma querda de 26,6 para 16,2 óbitos a cada 1.000 nascimentos, conforme pode se verificar no gráfico:

Tendência da taxa de mortalidade infantil (TMI) - Brasil e regiồes, de 2000 a 2010

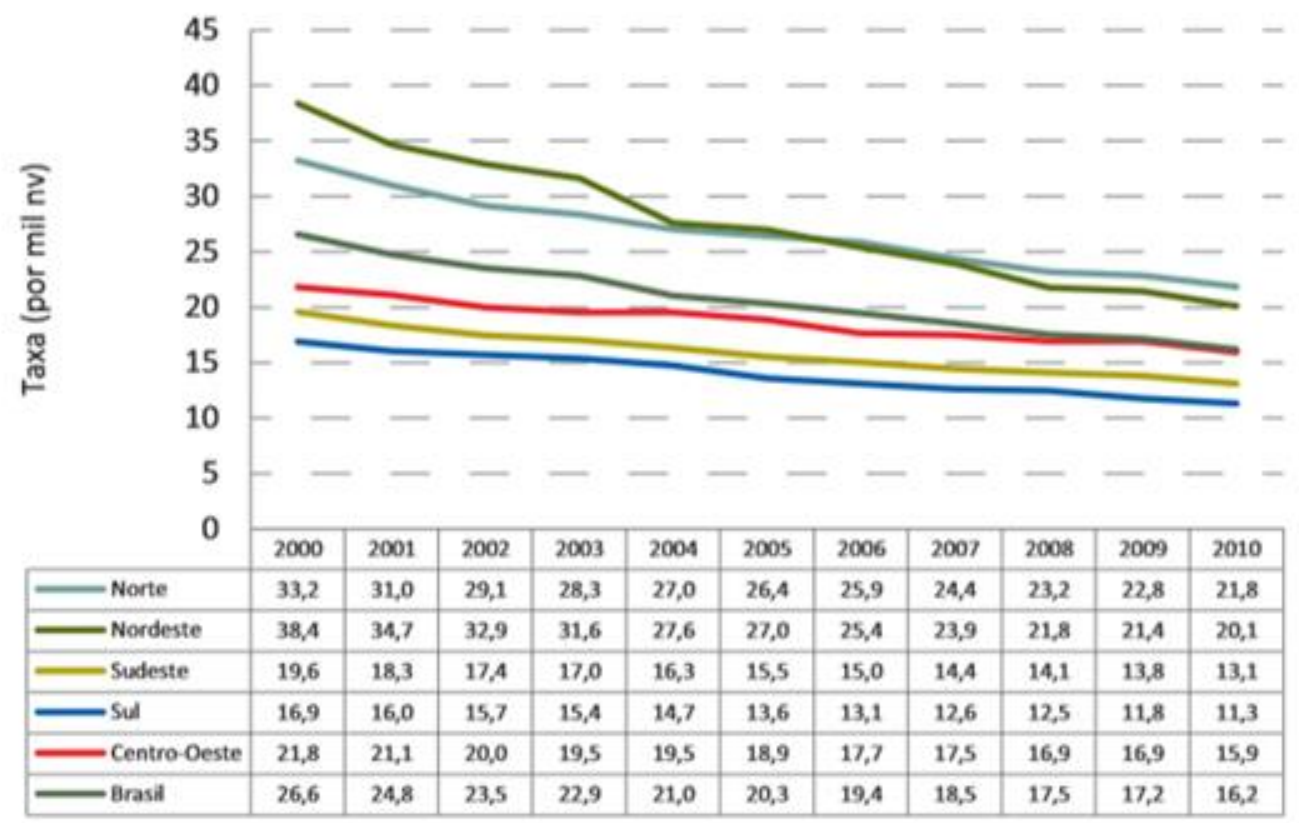

Fonte: CGIAE/DASIS/SVS/Ministério da Saúde.

Nota: estimativas com base nos dados sobre b́bitos do SIM e nascimentos do Sinasc corrigidos por fatores fornecidos pela pesquisa de busca ativa realizada em $2008^{4}$ 
Ademais, nota-se que a queda da mortalidade infantil ocorreu em todas as regiões do país, principalmente no Nordeste, cuja diminuição foi de quase $50 \%$, diminuindo de 38,4 para 20,1 mortes a cada 1.000 nascimentos. No entanto, o quantitativo de óbitos de bebês e recémnascidos principalmente, tem crescido na maioria das regiões brasileiras ou não tem apresentado melhora significante, conforme pode se verificar no gráfico a seguir:

\begin{tabular}{|c|c|c|c|c|c|c|c|}
\hline \multicolumn{7}{|c|}{ Mortalidade infantil segundo idade do óbito e principais causas.* } & \\
\hline \multicolumn{8}{|c|}{ Brasil e regiões, 1996-2010 } \\
\hline \multicolumn{8}{|c|}{ Óbitos infantis segundo idade de ocorrência (\%) } \\
\hline & Período & Norte & Nordeste & Sudeste & Sul & C.Oeste & Brasil \\
\hline \multirow[t]{3}{*}{0 a 6 dias } & 1996-199 & 49,7 & 40,1 & 52,6 & 47,2 & 50,7 & 47,6 \\
\hline & $2002-200$ & 50,2 & 50,1 & 51,9 & 49,1 & 50,8 & 50,7 \\
\hline & $2008-201$ & 52,2 & 55,8 & 50,8 & 51,1 & 51,0 & 52,7 \\
\hline \multirow[t]{3}{*}{7 a 27 dias } & 1996-199 & 12,0 & 11,5 & 13,4 & 12,7 & 13,4 & 12,6 \\
\hline & $2002-200$ & 13,6 & 12,8 & 17,3 & 16,4 & 17,4 & 15,1 \\
\hline & 2008-201 & 14,4 & 13,9 & 18,1 & 18,1 & 16,8 & 16,1 \\
\hline \multirow[t]{3}{*}{28 a 364 dias } & 1996-199 & 38,3 & 48,4 & 33,9 & 40,2 & 35,9 & 39,8 \\
\hline & $2002-200$ & 36,2 & 37,1 & 30,8 & 34,5 & 31,8 & 34,2 \\
\hline & 2008-201 & 33,4 & 30,3 & 31,1 & 30,8 & 32,1 & 31,2 \\
\hline \multirow[t]{3}{*}{$\begin{array}{l}\text { Obitos neonatais entre os } \\
\text { óbitos infantis (\%) }\end{array}$} & 1996-199 & 61,7 & 51,6 & 66,1 & 59,8 & 64,1 & 60,2 \\
\hline & $2002-200$ & 63,8 & 62,9 & 69,2 & 65,5 & 68,2 & 65,8 \\
\hline & 2008-201 & 66,6 & 69,7 & 68,9 & 69,2 & 67,9 & 68,8 \\
\hline \multirow[t]{3}{*}{$\begin{array}{l}\text { Afecções perinatais entre } \\
\text { os óbitos infantis (\%) }\end{array}$} & 1996-199 & 52,8 & 41,7 & 57,4 & 50,3 & 54,6 & 51,0 \\
\hline & $2002-200$ & 54,3 & 54,6 & 58,8 & 55,8 & 56,2 & 56,2 \\
\hline & 2008-201 & 56,2 & 61,1 & 59,1 & 57,9 & 56,1 & 59,0 \\
\hline
\end{tabular}

Fonte: MS/SVS/DASIS - Sistema de Informações sobre Mortalidade - SIM

${ }^{*}$ Classificadas segundo capítulos da CID- 10 .

Estudos realizados em 2010 no Brasil, demonstram ser grande o índice de mortalidade infantil ocorre no primeiro dia de vida do bebê, em que um a cada quatro mortes ocorreram nas primeiras 24 horas do pós-parto. Nesse diapasão, urge mencionar o grande quantitativo de prematuros que morrem no primeiro dia de vida, atingindo um percentual próximo de $70 \%$ do total de óbitos que ocorrem nas primeiras $24 \mathrm{~h}$ de vida, sendo as principais causas da morte súbita nesse primeiro momento da vida o pequeno peso com o qual a criança nasce e a questão da prematuridade. 
Distribuição dos óbitos infantis, segundo componentes - Brasil, 2010

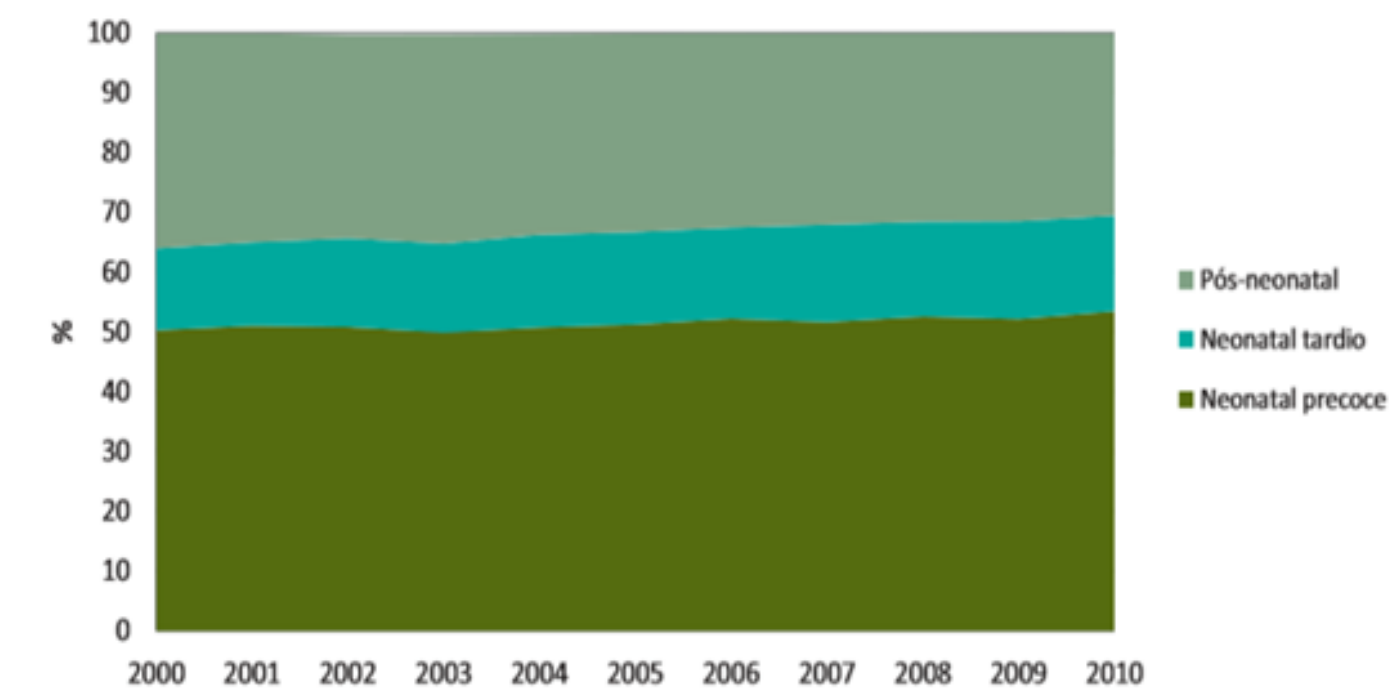

Fontes: CGIAE/DASIS/SVS/Ministério da Saúde, Sistema de Informaçōes sobre Mortalidade (SIM).

Verificou-se, ainda, que cerca de $80 \%$ desses bebês recém-nascidos possuíam menos de $2,5 \mathrm{~kg}$ e que $70 \%$ nasceram de forma prematura. Contudo, esse alto percentual de óbito no primeiro dia de vida com o peso adequado é preocupante, isto porque revela uma ausência de assistência médica adequada na gestação, parto e nascimento, que seria capaz de evitar essas mortes.

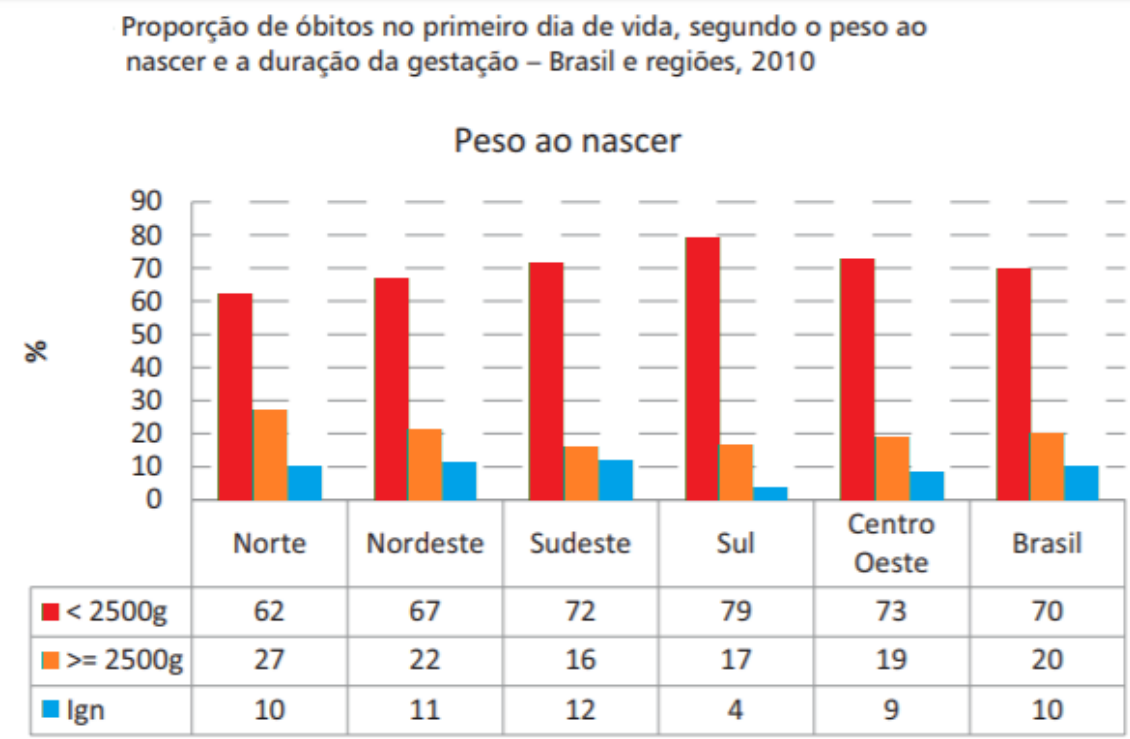




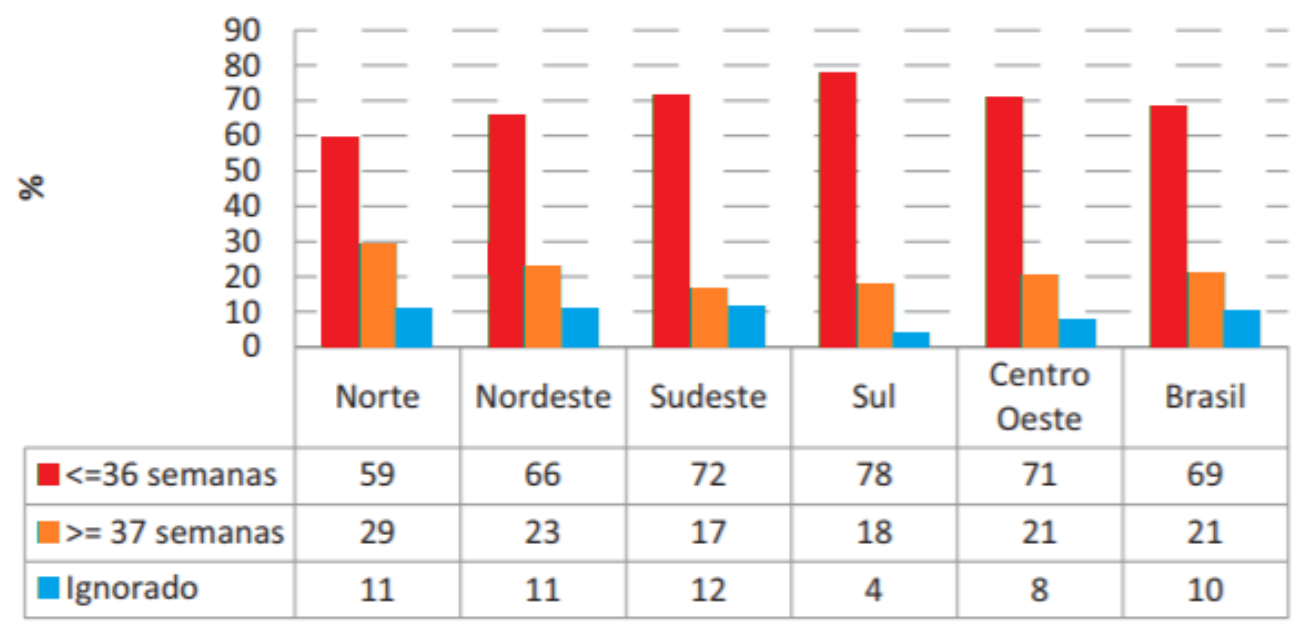

Fontes: CGIAE/DASIS/SVS/Ministério da Saúde, Sistema de Informaçōes sobre Mortalidade (SIM).

Conforme comentado, não existe uma análise bem aprimorada que defina as causas da SMSI, contudo, tem-se apontado que a prematuridade, que ocasiona retardo na maturidade do tronco cerebral e do sistema nervoso central, responsável pelo controle da respiração, batimentos e pressão arterial do bebê, com principal causa da SMSI, principalmente porque crianças com tendência a desenvolver a síndrome, possui dificuldade de acordar mesmo diante de uma situação que o cause estresse corporal, como é o caso da asfixia, pouco ou muito calor.

Ainda, sugere-se uma etiologia multifatorial acerca das causas da SMSI, isto porque estudos mostram que cerca de $88 \%$ dos casos de morte súbita infantil envolve dois ou mais fatores. Aponta-se que que os bebês prematuros nascidos com fatores de risco perinatal, como o tabagismo, idade da mãe, irmãos vitimados pela mesma síndrome e o período do inverno, sejam suas as principais causas.

Nesse sentido, sendo indefinidos os motivos ocasionadores da SMSI, medidas preventivas têm se mostrado eficazes. Assim, preocupação com a forma com a qual a criança dorme, incentivar a sucção não nutritiva com o uso de chupetas, aconselhamento acerca da melhor forma de cuidar, agasalhar e alimentar o bebê; tem fortemente diminuído o número de casos relacionados com a síndrome. Dessa forma, os aconselhamentos de saúde preventiva e medidas educativas revela ser algo imperioso e comprovadamente eficaz. 
A etiologia dessa enfermidade ainda é demasiadamente obscura, o que revela a necessidade de se desenvolverem mais análises e estudos epidemiológicos específicos sobre a SMSI, para fins de verificação das causas e óbito e posterior prevenção destas, por intermédio de políticas públicas adequadas e específicas.

\section{Principais causas}

Ainda não existem estudos que delimitem categoricamente as causas da morte súbita infantil, razão pela qual estudiosos vêm buscando estudar fatores de risco variados que acreditam estar fortemente associado a SMSI. Alguns desses fatores não podem ser modificados, como é o caso da criança que nasce com uma má formação que não é passível de remediação, prematuridade e baixo peso; outros, podem ser evitados por meio de algumas atitudes preventivas.

Pode-se dividir as causas da morte súbita infantil em intrínsecas e extrínsecas ao lactente, sendo as primeiras relacionadas a idade, gênero, perfil genético, parentesco, prematuridade, exposição ao fumo e fatores socioeconômicos em geral; e o segundo grupo envolve questões sobre a forma que o bebê dorme, hiperaquecimento, partilha de cama com terceiros, características do local onde é colocado para dormir e desenvolvimento de enfermidades, em especial as infecciosas (LOPES, 2013/2014, p. 24-31).

Quanto as causas intrínsecas à criança, verifica-se que a síndrome acomete com mais frequência crianças do sexo masculino do que feminino, em uma proporção aproximada de 3:2,5, que a incidência é ligeiramente maior em bebês prematuros, com baixo peso e cujas mães possuem menos de 20 anos. Ainda, afere-se que cerca de $90 \%$ dos casos da síndrome ocorrem, em geral, entre o primeiro e o sexto mês da vida do bebê, havendo mais registros de morte entre o segundo e o quarto mês. Outrossim, os índices de SMSI também possui variação de acordo com a etnia da criança, sendo mais comum em crianças negras não-hispânicas e índios americanos, é bem mais elevada do que nos outros grupos étnicos (OLANREWAJU; MOON, 2008, p. 31-39).

Ademais, correlacionam-se os fatores socioeconômicos ao risco de morte, isto porque o desenvolvimento dessas doenças a ausência de quantidade suficiente de cobertores e agasalhos colocados pela mãe no bebê, bem como de uma alimentação adequada. Logo, aponta-se, que o 
maior número de mortes subidas infantis é encontrado na população de baixa renda, sendo essa a que possui menor grau de escolaridade, bem como menor condição financeira (SPENCER; LONGAN, 2004, p. 366-373) para adquirir um enxoval, acessórios, bem como alimentos, que atendam a integralidade das necessidades do bebê.

Ainda sobre os fatores intrínsecos, Shat et al (2006), revelou que o tabagismo também é fator ocasionador de grande quantitativo de mortes súbitas infantis, isto porque gestantes que fumam durante o período gestacional ou que fumam após a gravidez, colocando o bebê na posição de fumante passivo, aumenta o risco da incidência da síndrome. O contato precoce da criança com a fumaça e demais toxinas, interfere no funcionamento cardiovascular e respiratório do bebê, tornando-o mais suscetível a episódios de sufocamento ou asfixia. Estimase que aproximadamente $21 \%$ dos casos poderiam ter sido evitados caso as mães não fumassem nem durante nem após a gravidez, o que indica a importância das políticas informacionais acerca dos malefícios do fumo para as gestantes.

Quanto ao grupo de causas extrínsecas, umas das grandes causas de risco de SMSI envolve a posição a qual o bebê dorme, isto porque estudos apontam que quando esta dorme na posição lateral são maiores a possibilidades do bebê rolar para a posição ventral do que para a posição dorsal (SCRAGG; MITCHELL, 1998), e, então, morrer asfixiado.

Atestou-se que sazonalidade também é fator ocasionador do aumento da morte súbita, isto porque verificou-se que no inverno há um maior quantitativo de SMSI, e isto se deve ao fato de ser ambiente mais propício ao desenvolvimento de doenças que se desenvolvem mais facilmente nos períodos frio do ano como hipotermia; doenças respiratórias virais, como influenza, bronquite, bronquiolite; deficiências bioquímicas, que causam desnutrição, anemia e desidratação; dentre outras (VICTORA ET AL, 1987, p. 490-496), bem como em razão de ser mais fácil a morte do bebê por hipotermia (OLANREWAJU; MOON, 2008, p. 31-39).

A partilha da cama com terceiros também consiste em causa da morte súbita infantil, e esta está intimamente relacionada a facilidade dos pais, ou terceira pessoa, rolar sobre o bebê enquanto dorme, matando-o asfixiado, devendo, então ser algo a ser evitado. Além disso, a superfície em que a criança dorme, bem como os objetos que são colocados perto, podem influenciar no aumento do risco da morte súbita, pois superfícies muito moles, almofadas e brinquedos pode provocar o sufocamento ou aprisionamento do lactente.

Da mesma forma que a ausência de cobertores suficientes pode causar a hipotermia, o excesso pode causar o hiperaquecimento do bebê, levando-o a morte. Assim, cobrir a cabeça 
da criança, local de maior perda de calor pelo lactante, com cobertores ou outros objetos, tem dado causa ao aumento do risco, em razão de proporcional a hipoxia e hipercapnia, situações que afetam o controlo autonômico do lactente.

As causas de óbitos infantis acima elencadas, revelam a necessidade e importância da devida assistência médica e social, conferindo mais orientações de saúde preventiva, relacionadas ao parto, ao recém-nascido e à criança no primeiro ano de vida.

\section{Possíveis formas de prevenção}

Estudos realizados por membros da American Academy of Pediatrics, elencaram as principais recomendações, bem como importância dessas, a serem dadas as gestantes para fins de evitar a incidência da Síndrome da Morte Súbita Infantil, quais sejam: (i) bebês prematuros; (i) bebês com menos de 4 meses; (iii) pais fumantes; (iv) uso de bebidas alcoólicas ou medicamentos sedativos durante a gestação; (v) uso de cama compartilhada com terceiros, sofás, poltronas, travesseiros, colchas ou seja, coisas que facilitem a asfixia da criança; (vi) ausência de agasalho adequado nos dias frios; (vii) uso de chupeta, isto porque estudos têm demonstrado que seu uso colabora para prevenir a morte súbita; (viii) não expor o lactente a cigarro; (ix) evitar hiperaquecer a cabeça do lactente; (x) pré-natal adequadamente realizado; (xi) imunização para controle e prevenção de enfermidades; e (xii) colocá-lo para dormir em posição prona.

Dentre essas, a posição em que se coloca o bebê para dormir, o local que se coloca a criança para dormir, o adequado agasalhamento, o aleitamento materno e o uso de chupetas, têm sido as orientações que mais carecem de atenção, em razão da asfixia, das doenças que se desenvolvem mais facilmente no frio e da desnutrição, serem as principais causas da Síndrome da Morte Súbita Infantil.

A posição e o local que a criança dorme, tem se mostrado ser importante fator para fins de evitar a morte súbita, isto porque verificou-se que colocá-lo na posição supina consiste na melhor escola, isto porque são menores as chances do lactente virar para baixo, provocando sua asfixia. Somado a isso, é importante que o local seja um colchão de superfície firme coberto com apenas um lençol, pois isso evitará que ele morra sufocado com o mesmo ar. Inclusive, 
havendo irmãos gêmeos, é importante que devem dormir em locais separados, para evitar o risco de um rolar sobre o outro.

Trata-se de fator tão importante que, em 1991, criou-se na Inglaterra o chamado Back to sleep, para fins de divulgar a importância dos bebês dormirem para cima, tendo em vista que a posição dorsal facilita que ele vire para baixo e, assim, tenha uma morte silenciosa por falta de ar (JONES, 2004, p. 10-20). Com a aplicação de medidas associadas a posição do sono do bebê, houve a comprovação da eficácia dessas, tendo em vista a redução significativa da mortalidade por essa razão. A mesma campanha, quando lançada nos Estados Unidos, reduziu em $50 \%$ a ocorrência da síndrome.

A amamentação é apontada como outra forma de prevenção da SMSI, devendo ser mantida até, no mínimo, os seis meses do bebê, pois é através desse processo que a criança obtém os nutrientes necessários, assim como anticorpos, por meio do leite materno. Nesse sentido, aleitamento colabora com o desenvolvimento e crescimento, bem como configura fator imunoregulatório, isto porque possui diversas enzimas bioativas, hormônios, agentes imunológicos, dentre outros (GOLDMAN ET AL, 1996, p. 251-258). Contudo, é importante citar que não consiste em prática decisiva para a vida do lactente, uma vez que alimentá-lo com outras substâncias ainda é mais eficiente do que nenhum alimento.

Por fim, existem pesquisas que indicam que o uso de chupetas tem se revelado um método eficaz para fins de evitar a morte súbita infantil, isto porque o uso de chupeta previne que a língua impeça a passagem de ar, reduz os fluxos gastroesofágicos, ajuda no desenvolvimento da respiração bucal e ajuda a função respiratória.

O uso do acessório, em regra, é recomentado até o bebê completar 365 dias, sendo esse o período de maior risco de incidência da SMSI, e que, coincidentemente, trata-se de fase em a criança precisa de mais sucção. Estudos concluíram que o uso da chupeta pode alterar o controle cardíaco autônomo durante períodos de sucção e não sucção, ajudando na regulação do controle cardíaco autônomo, que pode ser uma forma de prevenir a morte súbita da criança. Além disso, a chupeta colabora do desenvolvimento da respiração bucal, para casos de obstrução das vias aéreas. (COZZI, ALBANI, CARDI; 1979, p. 329-338). Assim,

Isto exposto, resta imperioso que os profissionais de saúde devem se posicionar no sentido de divulgar essas recomendações durante o pré-natal e após o parto; a mídia deve divulgar essas medidas preventivas e o poder público deve desenvolver campanhas informativas e preventivas, para fins de diminuir o risco da incidência da Síndrome da Morte Súbita Infantil. 


\section{Considerações finais}

Estudos realizados em outros países já haviam identificados causas semelhantes da Síndrome de Morte Súbita Infantil, apontando a prematuridade, do fumo e consumo de álcool pela mãe como causa de malformações, desnutrição, posição em que o bebê dorme, infecções, hipotermia e hipertermia, como as principais causas.

Apesar de uma diminuição da taxa de mortalidade infantil no Brasil, o quantitativo referente as mortes de bebês menores de 1 ano de idade continua elevada quando comparada a de outros países. Logo, o referido alto quantitativo de mortes no primeiro ano de vida da criança revela a necessidade de ampliar o acesso e melhoria dos serviços de saúde, com o escopo de possibilitar uma atenção melhor à gestante durante o pré-natal e no período pós-parto.

Conforme comentado, raros são os estudos acerca do tema, razão pela qual resta imperioso a análise e uniformização de uma epistemologia no Brasil, para que seja possível a avaliada correta da doença, para fins de melhorar as formas de prevenção. Ainda, a necessidade de desenvolvimento políticas educacionais é clarividente, isto porque a falta de conhecimento e ausência da devida orientação é fator que aumenta os riscos de ocorrência da SMSI.

Assim, é preciso a implementação de políticas intervencionistas de curto prazo para fins de orientação de ações mais fácies de serem atingidas, como ensinar a melhor posição que o bebê deve dormir, bem como a melhor forma de organizar o local adequado para tanto, a importância da amamentação, as complicações causadas pelo tabagismo etc; guardando para as políticas públicas de longo, ações que visem a melhora socioeconômica como um todo.

A SMSI pode ser evitada, mas, para tanto, é preciso que o Poder Público desenvolva ações educativas direcionadas as gestantes, mães, pais e cuidadores. Assim, é notório que o combate a Síndrome da Morte Súbita Infantil exige um elevado grau de comprometimento por parte do gestor público em todas as esferas de poder, com fulcro de alcançar uma maior qualidade nos serviços de saúde direcionados a gestante na rede pública de saúde. Nesse diapasão, deve-se buscar melhorar o quadro atual por meio da promoção, proteção e recuperação da saúde pública, observadas as peculiaridades de cada local, com a finalidade de melhorar a qualidade da rede de saúde para as gestantes e mães e, consequentemente, reduzir os casos de mortalidade subida infantil. 


\section{Referências}

BARROS, F. C.; VICTORA, C. G.; TEIXEIRA, A. M. B.; PUERTO FILHO, M. Mortalidade perinatal e infantil em Pelotas, RS: nossas estatísticas são confiáveis? Cad. Saúde públ, 1:348$58,1985$.

BARSMAN, S.G.; DOWLING, D.A.; DERMATO, E. G.; CZECK, P. Neonatal Nurses' Beliefs, Knowledge, and Practices in Relation to Sudden Infant Death Syndrome RiskReduction Recommendations. Advances in Neonatal Care. Vol 15, n 3, p. 209-219, 2015. Disponível em: < https://www.semanticscholar.org/paper/Neonatal-nurses'-beliefs\%2Cknowledge $\% 2 \mathrm{C}$-and-practices-BarsmanDowling/3b99df5b17c261de8fc412c13216948efd77b92b>. Acesso em: 13/05/2018.

BEZERRA, J. L. O.; BEZERRA, M. A. L.; CARVALHO, K. M.; MOURA, T. H. M.; NOVAES, L. F. G.; PEDROSA, L. Fatores associados ao conhecimento das mães sobre a Síndrome da Morte Súbita do Lactente. Escola Anna Nery de Enfermagem. Vol. 19, n 2, p. 303-309. 2015. Disponível em: < http://www.scielo.br/pdf/ean/v19n2/1414-8145-ean-19-020303.pdf>. Acesso em: 13/05/2018.

BECKWITH, J. B. Observations on the pathological anatomy of the sudden infant death syndrome. Seattle: University of Washington Press; 1970.

COZZI, F.; ALBANI, R.; CARDI, E. A common pathophysiology for sudden cot death and sleep apnoea. The vacuum-glossoptosis syndrome. Med Hypotheses Magazine. Vol. 5, $\mathrm{n}^{\mathrm{o}}$ 3, $\mathrm{p}$. 329-338, março. 1979. Disponível em: <https://www.ncbi.nlm.nih.gov/pubmed/223019>. Acesso em: 13/05/2018.

FERNANDES, A.; FERNANDES, C.A; AMADOR, A.; GUIMARÃES, F. Síndrome da morte súbita do lactente: o que sabem os pais? Acta Pediátrica Portuguesa. 2012; 43(2): 59-62. Disponível em: http://www.spp.pt/Userfiles/File/App/Artigos/33/20121029175104_ ArtOriginal_FernandesA_43.pdf. Acesso em: 14/05/2018.

GOLDMAN, A.S.; CHHEDA, S.; GAROFALO, R.; SCHMALSTIEG, F.C. Cytokines in human milk. Properties and potential effects upon the mammary gland and neonate. $J$ Mammary Gland Biol Neoplasia. 1996.

GUTIERREZ, C.A. Síndrome de la muerte súbita del lactante. Medicina de Família.net. 2001 Disponível em: <http://www.zonadesalud.org>. Acesso em: 10/05/2018.

JONES, W.M. Supine and prone infant positioning: a winning combination. The Journal of Perinatal Education. Vol. 13, $\mathrm{n}^{\mathrm{o}}$ 1, p; 10-20. 2004. Disponível em: < https://www.ncbi.nlm.nih.gov/pmc/articles/PMC1595182/>. Acesso em: 11/05/2018. 
LOPES, T. F. N. Síndrome da morte súbita do lactente. 2013/2014. Dissertação (Mestrado em Medicina). Mestrado Integrado de Medicina. Universidade do Porto, Instituto de Ciências Biomédicas Abel Salazar, Porto.

MCVEA K.L.; TURNER, P.D., PEPPLER, D.K. The role of breastfeeding in sudden infant death syndrome. $J$ Hum Lact. Vol. 16, p. 13-20. 2000. Disponível em: < http://citeseerx.ist.psu.edu/viewdoc/download?doi=10.1.1.908.9270\&rep=rep1\&type=pdf >. Acesso em: 13/05/2018.

MINISTÉRIO DA SAÚDE. Secretaria de Vigilância em Saúde. Departamento de Análise de Situação de Saúde. 2012. Departamento de Informática do SUS - DATASUS. Informações de Saúde (TABNET). Estatísticas Vitais. Disponível em:< http://www2.datasus.gov.br/ DATASUS/index.php?area $=0205 \& V O b j=h t t p: / / t a b n e t . d a t a s u s . g o v . b r /$ cgi/deftohtm.exe?sim/cnv/inf10>. Acesso em: 14/05/2018.

MINISTÉRIO DA SAÚDE. Secretaria de Vigilância em Saúde/MS. Mortalidade infantil no Brasil: tendências, componentes e causas de morte no período de 2000 a 2010 in Saúde Brasil 2011: uma análise da situação de saúde e a vigilância da saúde da mulher. 2011. Disponível em:

http://repositorio.unb.br/bitstream/10482/12478/1/CAPITULO_MortalidadeInfantilBrasil.pdf. Acesso em: 11/05/2018.

OLANREWAJU, O.; MOON, Y. R. Sudden infant death syndrome: a review of literature. Current Pediatrics Reviews, 17(4), 31-39. 2008.

OLIVEIRA FILHO, P. M.; JARDIM, P. T. C.; ROCHA, M. C. L.; SOVIEIRO, V.; CRUZ, R. A. Importância da amamentação no desenvolvimento da criança saudável. Conhecimento básico para o cirurgião-dentista. Revista Arquivo brasileira odontologia. Vol. 4, no 2, p. 76 80.2008 .2 Disponível em: $<$ http://periodicos.pucminas.br/index.php/Arquivobrasileirodontologia/article/view/1265/1327 >. Acesso em: 13/05/2018.

PUFFER, R. R. \& SERRANO, C. V. Patterns of mortality in childhood. Washington, DC, Pan American Health Organization, 1975.

VICTORA, C. G.; BARROS, F. C. As mães lembram o peso ao nascer de seus filhos? Rev Saúde Pública. 1985;19(3):195-200. http://dx.doi.org/10.1590/S0034-89101985000300001.

VICTORA, C. G.; NOBRE, L. C.; LOMBARDI, C.; TEIXEIRA, A. M. B.; FUCHS, S. M. C.; MOREIRA, L. B.; GIGANTE, L. B.; GINGANTE L. P.; BARROS, F. C. Quadro epistemológico das mortes súbitas na infância em cidades gaúchas (Brasil). Revista de Saúde Pública. São Paulo, vol. 26, $\mathrm{n}^{\mathrm{o}}$ 6, p. 490-496, 1987. Disponível em: < www.scielo.br/scielo.php?script=sci_abstract\&pid $=\mathrm{S} 0034$ -

89101987000600004\&lng=e\&tlng=en>. Acesso em: 13/05/2018.

ROSSI, A.; MESQUITA, K. S. F.; ROSSI, R.; QUEIROZ, A. M. Possível papel de chupetas na prevenção da síndrome da morte súbita na infância. Revista da Faculdade de Odontologia 
de Lins/Unimep. Vol. 24, $\mathrm{n}^{\circ}$ 1, p. 51-57, janeiro/junho. 2014. Disponível em:<https://webcache.googleusercontent.com/search?q=cache:1eD7HVrhqYwJ:https://www. metodista.br/revistas/revistas-

unimep/index.php/Fol/article/download/1847/1347+\&cd=1\&hl=pt-BR\&ct=clnk\&gl=de .

Acesso em: 13/05/2018.

SCRAGG, R.K.; MITHCELL, E. A. Side sleeping position and bed sharing in the sudden infant death syndrome. Annals of Medicine. vol. 30, $\mathrm{n}^{\mathrm{o}}$ 4, 345-349, 1998.Disponível em: < https://www.researchgate.net/publication/13502189_Side_sleeping_position_and_bed_sharin g_in_the_sudden_infant_death >. Acesso em: 13/05/2018.

SHAH, T.; SULLIVAN, K.; CARTER, J. Sudden infant death syndrome and reportes maternal smoking during pregnancy. American Public Health Association. Vol. 96, $\mathrm{n}^{\circ}$ 10, p. 1757-1759. 2006. Disponível em: https://www.ncbi.nlm.nih.gov/pmc/articles/PMC1586150/>. Acesso em: $11 / 05 / 2018$.

\section{Como citar este artigo (Formato ABNT):}

MARTINS, Maria Eliana P.; AMORIM, Liromaria Maria de; RODRIGUES, Micaelle Nayara D.; LIMA, Danielly G. S.; MOREIRA, Jamacir F. Síndrome da Morte Súbita Infantil (SMSI): aspectos acerca das principais causas e as formas de prevenção. Id on Line Rev.Mult. Psic., 2018，vol.12, n.41, p.192-205. ISSN: 1981-1179.

Recebido: 07/06/2018

Aceito 14/06/2018 